\section{El desarrollo local, una aproximación conceptual}

José Arocena

Docente investigador de grado y posgrado. Universidad Católica de Uruguay. Miembro del Consejo Directivo del Programa Desarrollo Local del Centro Latinoamericano de Economía Humana (CLAEH). Consultor de organismos internacionales: OCDE, BID, Unión Europea, OIT, PNUD.

\section{1. ¿Qué es lo "local"?}

Referirse a lo "local" no está exento de ambigüedad, de imprecisiones, de dificultades de definición. Las preguntas que surgen y que frecuentemente no encuentran respuestas convincentes es: ¿qué es lo local? ¿Se trata de una escala que habría que precisar en número de habitantes o en kilómetros cuadrados? ¿Se refiere a una unidad político-administrativa que abarca una parte del territorio nacional? ¿Supone un sistema social con cierta autonomía?

La única forma de definir lo "local" es referirlo a su noción correlativa lo "global". Cuando algo se define como "local" es porque pertenece a lo "global". Así, un departamento o una provincia es local con respecto al país global, y una ciudad es local con relación al departamento o a la provincia. La noción de "local" es, por lo tanto, relativa. Es por eso que un proceso de desarrollo local sólo puede ser analizado en cuanto a la sociedad global en la que está inscrito.

Si nos detenemos, por ejemplo, en los procesos de desarrollo local en Europa y en América Latina, vamos a encontrar importantes diferencias en la medida en que se inscriben en sociedades con un desarrollo histórico y una estructura socioeconómica claramente diferenciadas. Una manera de visualizar mejor esas diferencias es recordar rápidamente cómo surgen estos procesos en uno y otro continente.

\section{2. ¿Cómo surge el tema?}

\subsection{En Europa}

Se suele decir que en los países industrializados la cuestión del desarrollo se consideró como una problemática propia a partir de la crisis que se inició a comienzos de la década del setenta. Al cabo de un período de crecimiento acelerado y sostenido, los países llamados "desarrollados" conocieron la recesión y la crisis. Las poblaciones de estos países, acostumbradas a un aumento
Desarrollo local y regional /

Perspectivas

\begin{abstract}
Este artículo para la Universidad Nacional del Litoral, me fue solicitado con la idea de problematizar los conceptos de desarrollo local y regional, brindando un panorama respecto de las discusiones teóricas, conceptuales y políticas sobre los alcances de estas nociones, que apunten de esta manera, a interpelar tanto la posición de las universidades públicas (como actores relevantes), como las intervenciones que se dan en el marco de la extensión. Lo que propongo a continuación, son algunas reflexiones sobre las nociones que me parecen fundamentales en esta temática: lo "local", el surgimiento del tema del desarrollo local, la sociedad local y el actor local. Estas nociones se han trabajado teóricamente en los últimos treinta años.
\end{abstract}

constante de su capacidad de consumo, tuvieron que aceptar una cierta disminución de su poder adquisitivo. Las inversiones cayeron de manera importante, los aparatos industriales protagonistas del crecimiento perdieron sus márgenes de utilidades y procedieron a despidos masivos. Se intentaron todas las fórmulas; se ensayaron todas las políticas económicas. Se lograron éxitos parciales, pero la desocupación mantuvo sus índices alarmantes y generó fenómenos crecientes de exclusión social.

Fue frente a esta situación que las sociedades "desarrolladas" buscaron otras formas de movilización del potencial humano, mediante el estímulo de la pequeña iniciativa de carácter casi siempre local. Fundamentalmente, se propició desde las políticas estatales, la creación de pequeñas y medianas empresas en aquellas zonas que habían sido más perjudicadas por la crisis. Se desarrollaron programas especiales de formación para la creación y la gestión empresarial, se promovió el crédito en condiciones ventajosas, se crearon asociaciones de creadores de pequeñas empresas. El objetivo fue resumido en la consigna que pronunció un primer ministro francés: "que cada uno cree su empleo".

La inserción local de las pequeñas empresas llevó progresivamente a considerar la dimensión local del desarrollo. Particularmente en Francia, España e Italia, se comenzó a plantear lo que se llamó procesos de "desarrollo local" o de "desarrollo regional". Las viejas identidades locales y regionales europeas se expresaron entonces no solo a nivel cultural, sino también a nivel socioeconómico.

En el caso europeo, los procesos de desarrollo local se basaron fuertemente en esas viejas identidades, que actuaron como motores de la generación local y regional de riqueza.

\subsection{En América Latina}

En las últimas tres o cuatro décadas, varios países de América Latina asistieron a la emergencia de un fenómeno que se ha llamado a veces "nuevos movimientos sociales". Se trataba más bien de formas de autoorganización en función de reivindicaciones 


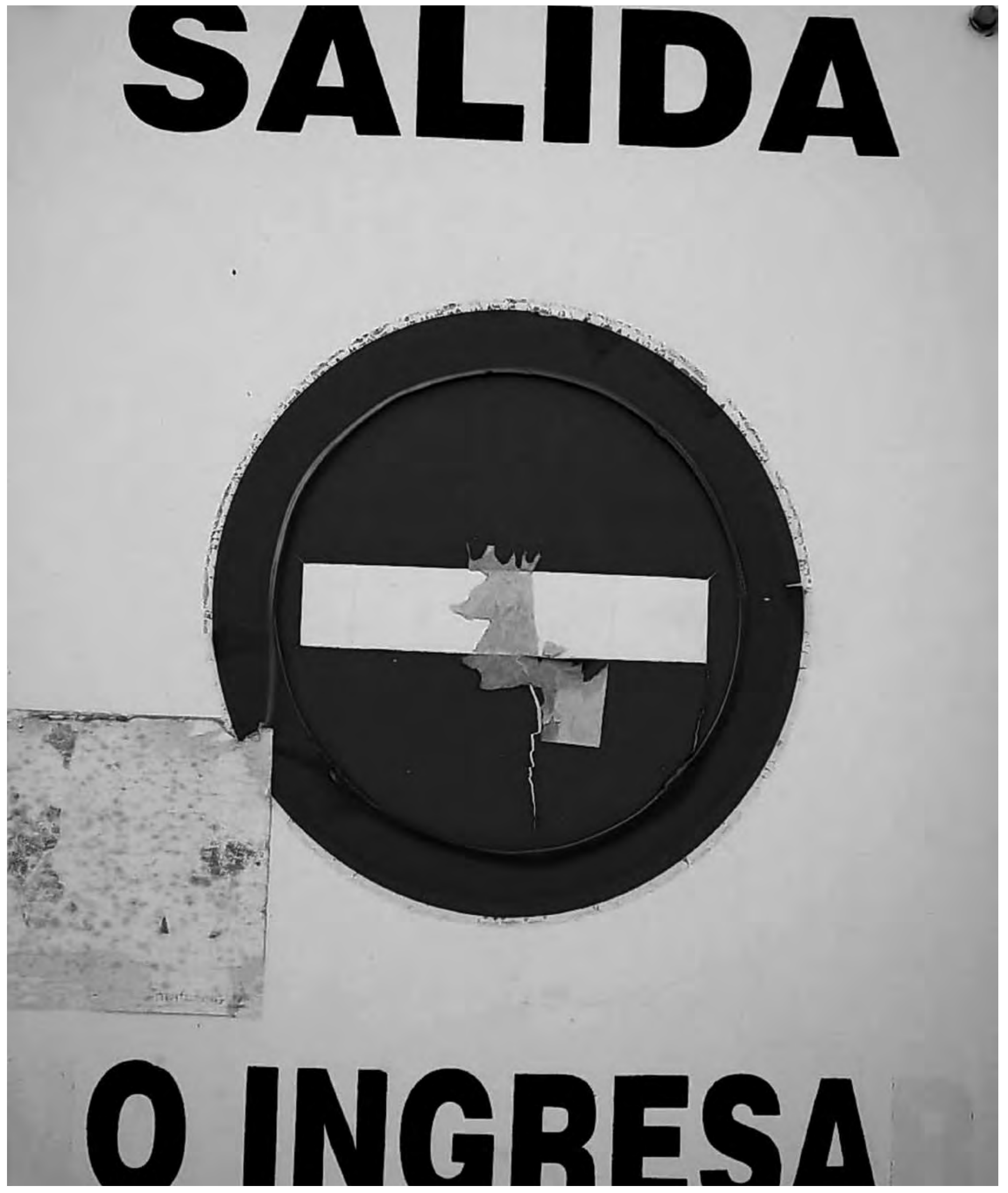


colectivas en el área de la vivienda, la salud, la alimentación, el medio ambiente, los servicios públicos o el consumo en general. El barrio o la pequeña ciudad constituyeron el lugar natural de expresión colectiva a causa de las relaciones directas entre los individuos frente a las exigencias de la vida cotidiana Las causas de la emergencia de estas organizaciones no fueron solamente socioeconómicas (degradación del nivel de vida) sino, también, políticas y culturales. Ha habido al mismo tiempo reivindicaciones con relación al nivel de vida, búsqueda de formas de participación sociopolítica y afirmación de ciertos valores como la solidaridad y la libertad.

En el origen de estas iniciativas se encuentran fundamentalmente dos tipos de actores: asociaciones de vecinos y organizaciones nacionales de la sociedad civil. Las primeras se constituyeron en función de reivindicaciones concretas (agua, saneamiento, alumbrado público) y frecuentemente trascendieron sus objetivos originales para convertirse en actores del desarrollo, al impulsar iniciativas comunitarias como huertas vecinales, talleres, servicios, etc. Las segundas, utilizando fondos provenientes de fundaciones o de gobiernos extranjeros, desarrollaron proyectos dirigidos tanto al área de los servicios urbanos como al de las iniciativas productivas. Se puso el acento en la capacidad de iniciativa y de propuesta de organizaciones barriales, cooperativas, empresas asociativas. Se subrayó la importancia de la sociedad civil, se promovieron las formas de organización comunitaria y de participación ciudadana como marco necesario para el desarrollo local.

Estas experiencias de autoorganización han producido un efecto de valorización del espacio local como un medio apto para el desarrollo de prácticas democráticas. Cuando los gobiernos autoritarios habían prohibido el funcionamiento de partidos políticos y sindicatos, lo "local" emergió como el único lugar donde la participación era posible. En ciertos países, el desarrollo de estas organizaciones alcanzó un grado de madurez importante.

\section{3. ¿Cómo definir una sociedad local?}

Los comienzos de la temática del desarrollo local destacan entonces la dimensión "creación de empresas" y "creación de empleos" en Europa, mientras que en América Latina se parte de reivindicaciones socioterritoriales y se afirma el carácter de canal de participación y de profundización de la democracia que tienen estos procesos. Pero en ambos casos se apela al actor local y a la sociedad local como agente y como ámbito privilegiado para el impulso de nuevos procesos de desarrollo. Es entonces necesario definir la sociedad local.

No pretendemos plantear aquí las distintas definiciones que han existido del término "sociedad". Nos limitaremos a señalar los elementos que confluyen para constituir ese "mínimo necesario" que permita hablar de "sociedad" y, en el caso que nos ocupa, de "sociedad local".

No incluiremos en esta definición de sociedad local la cuestión del tamaño del territorio. Esta omisión no es casual. Para definir una sociedad como "local", su dimensión en términos de número de habitantes o de kilómetros cuadrados de superficie no es una variable significativa. Estos aspectos cuantitativos pueden variar en forma muy importante de una sociedad local a otra. Por otro lado, no toda subdivisión de un territorio nacional es una sociedad local. Puede haber fragmentaciones físicas, políticas, administrativas, de una gran ciudad o de una nación que no correspondan a sociedades locales. Una sección judicial, un departamento, una provincia, son subdivisiones territoriales que no coinciden forzosamente con sociedades locales. Para que este término pueda aplicarse a una realidad local, se debe dar un cierto número de condiciones que se expresan en dos niveles fundamentales: socioeconómico y cultural.

A nivel socioeconómico, un conjunto humano puede ser llamado "sociedad local" cuando lo que está en juego en las relaciones entre los grupos es principalmente de naturaleza local. Dicho de otro modo, cuando la producción de riqueza (por mínima que sea) generada en el territorio es objeto de negociaciones entre los actores, se convierte así en la base principal del sistema local de relaciones de poder (Arocena, 2002).

En este primer nivel, para que exista sociedad local, debe haber riqueza generada localmente sobre la cual los actores locales ejerzan un control decisivo tanto en los aspectos técnicoproductivos como en los referidos a la comercialización. En estos casos, los grupos locales definen sus diferentes posiciones en función de la influencia de cada uno sobre cómo se utiliza la riqueza generada por la sociedad local. Se constituirá así una jerarquía social regulada por la mayor o menor capacidad de cada uno de sus miembros de influir en la toma de decisiones sobre la utilización de la riqueza. 


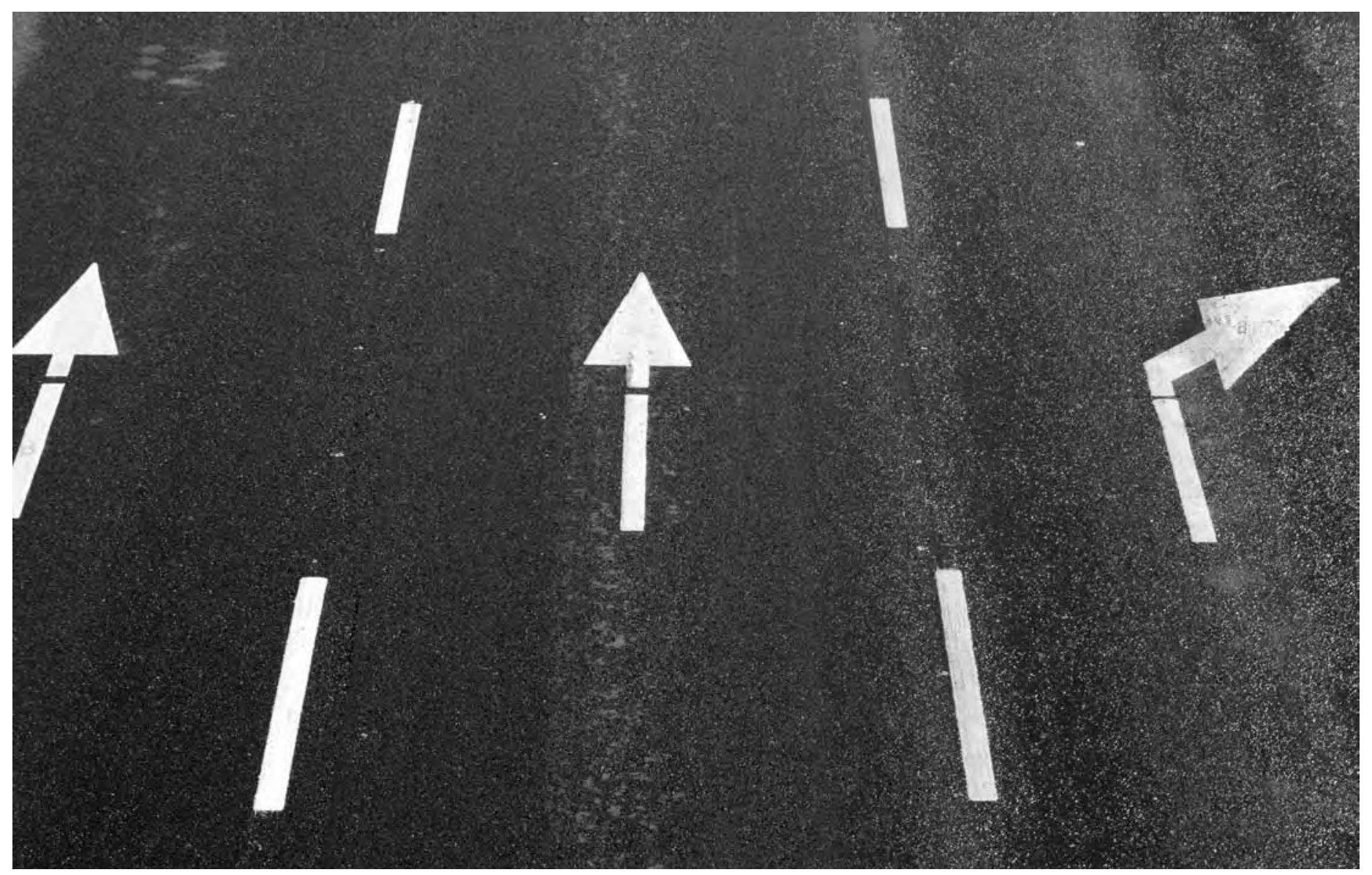

Pero esta dimensión socioeconómica no alcanza para definir una "sociedad local". Toda sociedad se nutre de su propia historia, constituyendo así un sistema de valores compartido por cada uno de sus miembros. Cada individuo se reconoce a sí mismo como formando parte de un conjunto bien determinado que puede identificarse con una ciudad, con un barrio de una metrópoli, con una región de un país, con una microrregión, etc. La expresión "yo soy de..." expresa pertenencia a una comunidad determinada. A este nivel cultural, la pertenencia se expresa en términos de identidad colectiva. Para que exista "sociedad local" es necesario que el conjunto humano que habita un territorio comparta rasgos comunes. Esto quiere decir que los individuos y los grupos constituyen una sociedad local, cuando muestran una "manera de ser" determinada que los distingue de otros individuos y de otros grupos. Este componente identitario encuentra su máxima expresión colectiva cuando se plasma en un "proyecto" común.

Un territorio con determinados límites, es entonces "sociedad local", cuando es portador de una identidad colectiva expresada en valores y normas interiorizados por sus miembros y cuando conforma un sistema de relaciones de poder constituido en torno a procesos locales de generación de riqueza. Dicho de otra forma, una sociedad local es un sistema de acción sobre un territorio limitado, capaz de producir valores comunes y bienes localmente gestionados.

\section{4. ¿Qué es actor local?}

En los procesos de desarrollo local, hay una dualidad básica que se expresa en la ecuación actor local-modelo de acumulación. Cuando la unidad de análisis es la sociedad local tomada desde su proceso de desarrollo, será necesario hablar de las estrategias de los actores locales y de las lógicas estructurales de la acumulación.

\subsection{Definición de actor local}

Una forma de definir un actor es relacionándolo con la escena social en la que desarrolla su acción. En este caso, nos estamos refiriendo a individuos, grupos o instituciones cuyo sistema de acción coincide con los límites de la sociedad local. En esta primera definición no se incluye la calidad de la acción, sino la escena en la cual se desarrolla. Así son actores locales los vecinos organizados que intentan mejorar la calidad de vida de un barrio, 
de una localidad, o de un área rural determinada; pero también son actores locales quienes se organizan para mantener y reproducir una determinada situación que produce destrucción de riquezas naturales y desestructuración de tejidos sociales.

Hay sin embargo otra forma de definir actor local.

Para Fernando Barreiro "los actores locales son simultáneamente motor y expresión del desarrollo local". Este mismo autor define tres categorías de actor local: a) los actores ligados a la toma de decisiones (político institucionales); b) los actores ligados a técnicas particulares (expertos profesionales); c) los actores ligados a la acción sobre el terreno (la población y todas sus expresiones activas). Barreiro subraya finalmente que el desarrollo local supone concertación, negociación, interacción entre actores (1988).

En este segundo tipo de definición al que tiende la caracterización de Barreiro, se liga la noción de actor local a la noción de desarrollo. Ello supone incluir en la definición no solamente la escena social en la que se desarrolla la acción, sino también el aporte que la acción realiza al desarrollo local. Importa la acción en sí misma, pero sobre todo importa el sentido que el actor imprime a la acción. Esto quiere decir que no todo individuo, grupo o institución actuando a nivel local puede ser considerado actor local del desarrollo o tal vez de manera más precisa, "actor-agente de desarrollo local". Esta distinción entre actor local (definido así porque actúa en la escena local) y agente de desarrollo local permite evitar confusiones en la terminología. Ubicándonos en esta definición más restrictiva:

"bajo la fórmula actor local, entendemos todos aquellos agentes que en el campo político, económico, social y cultural, son portadores de propuestas que tienden a capitalizar mejor las potencialidades locales. Es fundamental en esta definición el acento puesto en 'capitalizar mejor'. En efecto, se trata de buscar un mayor aprovechamiento de los recursos, pero destacando la calidad de los procesos en términos de equilibrios naturales y sociales" (Arocena, 2002).

Si se ligan entonces las nociones de "actor local" y de "desarrollo", dándole a la formulación "actor local" su sentido más fuerte (actoragente de desarrollo local) no puede entonces ser considerado como tal, una empresa que contamine con sus desechos industriales un río o que contribuya a deteriorar el medio ambiente en un área determinada. Tampoco será considerado actor local, aquel que en virtud de una lógica exógena, desarrolla acciones que producen una desarticulación de los tejidos sociales de la sociedad local. Es el caso, por ejemplo, de los enclaves turísticos construidos en el seno de sociedades agrarias, sin que sus miembros puedan apropiarse de los beneficios y controlar su desarrollo. Por último, es bien conocido el efecto pernicioso de ciertas "ayudas" al desarrollo, que desarticulan sistemas de producción locales, siendo incapaces de proponer alternativas viables.

El actor-agente de desarrollo local cuidará el equilibrio del medio natural, someterá las iniciativas de desarrollo al interés local, tratará de adaptar las tecnologías a las características de los sistemas locales de producción.

La generación de este tipo de actores-agentes de desarrollo local es una de las condiciones decisivas para el éxito de los procesos de desarrollo local. Las políticas de formación de estos agentes deberían ocupar un lugar de primera prioridad en todo planteo de planificación descentralizada.

\section{El modelo de acumulación}

La noción de actor local se expresa en una relación dual con la noción de "modelo de acumulación". No es posible entender uno sin el otro. El modelo de acumulación expresa un todo coherente, en el cual las mismas causas producirán siempre los mismos efectos. Si solamente tuviéramos en cuenta la dinámica del modelo, un componente cualquiera actuará en cualquier sociedad concreta de una manera constante. Todo será entonces previsible y generalizable. En esta lógica, los procesos de desarrollo local en una sociedad determinada, dependerán de la definición de las características del modelo de acumulación. Una vez definidas estas características, sería inútil imaginar que pueda haber márgenes de maniobra para las estrategias de los actores.

En las antípodas de la lógica del modelo se encuentra la lógica de la contingencia pura. Los procesos socioeconómicos tendrían un carácter totalmente imprevisible; las mismas causas podrían producir efectos contrarios en diferentes contextos. No sería entonces posible elaborar leyes explicativas de valor general ni reconocer ciertas regularidades estructurales.

¿Es posible escapar a la polaridad dilemática que significaría tener que optar entre la lógica del modelo y la lógica de la contingencia? ¿Es posible analizar un proceso de acumulación local como una realidad totalmente singular, pero que inscribe en sí misma ciertas regularidades estructurales?

Si la respuesta a estas preguntas es afirmativa, el análisis de los procesos de desarrollo local nos estaría planteando la necesidad de una concepción más precisa de las lógicas que existen al interior de un modelo de acumulación. En este sentido, parece muy claro el siguiente texto de Francisco Albuquerque:

"Entender esta cuestión creo que es clave para desbloquear el callejón sin salida en el que parecen encontrarse autores que niegan rotundamente el espacio posible de funcionamiento de las empresas de ámbito regional o local por el hecho de que la lógica estructural de la acumulación capitalista conducirá 


\section{6}

un territorio con determinados límites es, entonces, "sociedad local" cuando es portador de una identidad colectiva

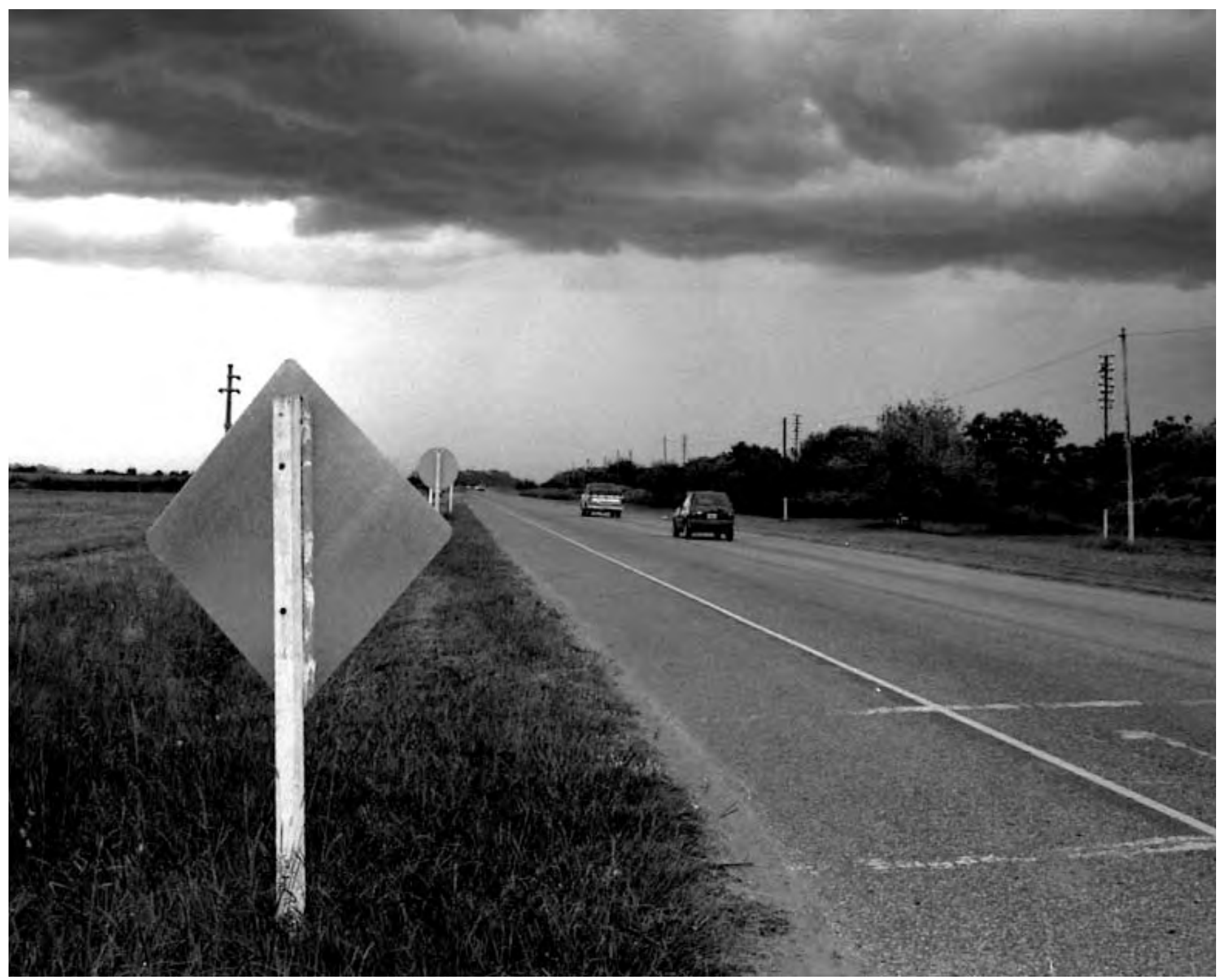




\section{6}

una sociedad local es un

sistema de acción sobre un

territorio limitado, capaz de

producir valores comunes y

bienes localmente gestionados

irreversiblemente a la monopolización y su integración en el único circuito económico mundial. Tal circuito único no existe. Será dominante pero no exclusivo. En mi opinión una cosa es la lógica genérica de la acumulación capitalista, cuestión que no se discute, y otra que dicha afirmación genérica refleje la más compleja realidad de la coexistencia de diferentes lógicas de acumulación concretas en el espacio de lo posible y con los constreñimientos dados en cada momento histórico" (Alburquerque, 1994).

Si se produce una coexistencia de diferentes lógicas de acumulación concretas, quiere decir que se está ubicando esta problemática fuera del campo de la racionalidad absoluta y sujeta, por lo tanto, a los efectos de la acción de los actores locales. En

otras palabras, estos procesos no están totalmente determinados por mecánicas preestablecidas de acumulación sino que admiten la idea de oportunidades al alcance de estrategias de actores capaces de utilizarlas.

Si bien no todo es estrategia porque, como acabamos de ver, el desarrollo local expresa también regularidades estructurales, la elaboración de estrategias locales ganadoras es un ingrediente esencial de estos procesos. El siguiente texto de Vázquez Barquero es muy explícito:

"En realidad, el carácter diferencial de la estrategia (de desarrollo económico local) es reconocer que el territorio también cuenta, que en el territorio se produce la coordinación/ descoordinación de las acciones de todos los agentes económicos y que, por lo tanto, la visión estratégica desde lo local es relevante para el desarrollo económico" (Vázquez Barquero, 1993).
En esta concepción, la globalización presenta no sólo amenazas sino también oportunidades para las regiones. Federico Bervejillo desarrolla la idea de la globalización como oportunidad:

"Solamente los territorios que han alcanzado ciertos niveles previos de desarrollo y que, además, cuenten con cierta masa crítica de capacidades estratégicas, pueden acceder a utilizar las nuevas oportunidades para su beneficio. En otras palabras, la globalización sería una oportunidad especialmente para territorios en niveles medios de desarrollo y dotados de capacidades estratégicas relevantes" (Bervejillo, 1995:34).

Las oportunidades no están al alcance de todos, suponen ciertas capacidades y determinados niveles de desarrollo. Pero lo que importa señalar es que al aceptar la idea de la relevancia de las estrategias locales se está abriendo —en teoría — la posibilidad de que toda sociedad local pueda jugar al interior de la estructura de acumulación capitalista. Su éxito dependerá sin duda de los factores y situaciones anotados por Bervejillo; pero en último análisis, esos factores pueden ser generados por políticas destinadas a crear las condiciones que permitan desarrollar las estrategias capaces de articular el potencial local con las oportunidades emergentes a nivel global.

Al abordar el tema de las estrategias estamos introduciendo, de hecho, uno de los condicionantes más importantes de los procesos de desarrollo local: la capacidad de generación de actores locales. Para algunos autores como Fernando Barreiro: "Los actores locales son simultáneamente motor y expresión del desarrollo local” (1998:88). Pero, como sucede con la categoría "modelo de acumulación", en este caso existe también un riesgo 
de reduccionismo, si le adjudicamos al actor local un potencial de acción más allá de los límites y restricciones del sistema. En realidad se trata de una interacción permanente entre actor y sistema. En este juego, el actor desarrolla sus márgenes de acción, ganando o perdiendo oportunidades, logrando disminuir las limitaciones que le vienen del sistema o, por el contrario, quedando más o menos paralizado por ellas.

Estas reflexiones sobre actor y sistema sólo tienen sentido si las relacionamos con un estado real de la sociedad. No se trata de simples categorías abstractas, sino de formas de pensar un momento de la historia en el que la afirmación de la posibilidad de márgenes de acción se constituye en una respuesta a la crisis de un modelo de integración social.

\section{Bibliografía}

Alburquerque, Francisco (1994). Apuntes sobre estrategia competitiva internacional y papel de las regiones. ILPES, Santiago de Chile.

Alburquerque, Francisco (2006). "Notas acerca del enfoque del desarrollo económico territorial para el empleo", en: SEBRAE, CORFO, OTCA. Desarrollo Económico Local, Red de desarrollo territorial y empleo para América Latina y el Caribe, Fortaleza.

Arocena, José (1995). El desarrollo local, un desafío contemporáneo. Primera edición: Nueva Sociedad, CLAEH y Universidad Católica del Uruguay, Caracas. Segunda edición ampliada: Taurus - Grupo Santillana - Universidad Católica del Uruguay. Montevideo, 2002.

Arocena, José (1998). Propuesta metodológica para el estudio de procesos de desarrollo local, CLAEH, Montevideo.

Arocena, José (2008). "El desarrollo local: los últimos 30 años". En: Revista Prisma, № 22, Universidad Católica del Uruguay.

Barreiro Cavestany, Fernando (1998). "Los agentes de desarrollo". En: Cuadernos del CLAEH, No 45-46, Montevideo.

Bervejillo, Federico (1995). "Nuevos procesos y estrategias de desarrollo.

Territorios en la globalización". En: Revista Prisma, № 4 Universidad Católica del Uruguay, Montevideo.

Marsiglia, Javier (1995). "Temas y actores en un escenario cambiante. La gestión social a nivel local”. En: Revista Prisma, № 4, Universidad Católica del Uruguay, Montevideo.

Marsiglia, Javier (2008). "Los gobiernos locales y las organizaciones de la sociedad civil: desafíos para la gestión concertada". En: Prisma, № 22, Universidad Católica del Uruguay, Montevideo.

Marsiglia, Javier (2009). Como gestionar las diferencias: la articulación de actores para el desarrollo local, Tesis de Maestría, Universidad Nacional de San Martín, Buenos Aires.

Marsiglia, Javier y Pintos, Graciela (1997). "La construcción del desarrollo local y regional: actores, estrategias y nuevas modalidades de intervención". En: Cuadernos del CLAEH, n 78/79, Montevideo.

Vázquez Barquero, Antonio (1993). Política económica local. Pirámide, Madrid. Vázquez Barquero, Antonio (1999). Desarrollo, redes e innovación. Pirámide, Madrid. 